\author{
Kinga Krawiec', Sylwia Dębska-Szmich², Urszula Czernek², Rafał Czyżykowski ${ }^{2}$, Piotr Potemski \\ ${ }^{1}$ Medical University of Lodz, Poland \\ ${ }^{2}$ The Department of Chemotherapy, Copernicus Memorial Multidisciplinary Centre for Oncology and Traumatology, Lodz; Chemotherapy Clinic, \\ Medical University of Lodz, Poland
}

\title{
Short bowel syndrome and severe skin toxicity as a complication of FOLFOX chemotherapy with panitumumab in a patient with colorectal cancer - a case report
}

\author{
Address for correspondence: \\ Kinga Krawiec \\ Klinika Chemioterapii Nowotworów \\ Uniwersytet Medyczny w Łodzi \\ WWCOiT im. M. Kopernika w Łodzi \\ ul. Pabianicka 62, 93-513 Łódź \\ Phone: 426895431 \\ Fax: 426895432
}

e-mail: kinga.krawiec@stud.umed.lodz.pl

Oncology in Clinical Practice 2019, Vol. 15, No. 6, 326-330 DOI: 10.5603/OCP.2019.0039

Translation: dr n. med. Dariusz Stencel Copyright @ 2019 Via Medica ISSN 2450-1654

\begin{abstract}
The combination of monoclonal antibodies targeting epidermal growth factor receptor (EGFR) with chemotherapy is the standard treatment in advanced colorectal cancer without mutations in the RAS and BRAF genes. We present a case of a 55-year-old female patient with unacceptable skin toxicity and short bowel syndrome caused by palliative FOLFOX chemotherapy combined with panitumumab. In 2012, after the emergence of an artificial anus due to gastrointestinal obstruction in the course of rectal cancer, the patient underwent inductive chemotherapy, preoperative chemoradiotherapy, and radical surgery. Tubular adenocarcinoma G2, ypT2N0 was diagnosed. In 2013 and 2015 she underwent two additional surgeries including intestinal resection due to obstruction of the gastrointestinal tract and enterovaginal fistula. In February 2018 she was qualified for palliative chemotherapy because of inoperable relapse. Due to very good performance status (PSO) and absence of mutations of RAS and BRAF genes, regardless of being underweight and suffering from loose stools persisting from the time of surgery, FOLFOX chemotherapy with panitumumab was introduced. After the second administration of drugs an acne-like rash, hand-foot syndrome, and diarrhoea appeared. Intensification of symptoms and manifestations of short bowel syndrome were observed afterwards. Topical treatment of skin lesions, doxycycline, and anti-diarrhoeal therapy were introduced, with a mediocre therapeutic effect. Imaging confirmed disease stabilisation, but due to the deterioration of both performance status and life quality, anti-cancer treatment was discontinued. This case draws attention to the necessity of caution while qualifying for potentially toxic combination chemotherapy. Key words: panitumumab, monoclonal antibodies, colorectal cancer, epidermal growth factor receptor, skin rash, short bowel syndrome, nutritional intervention
\end{abstract}

Oncol Clin Pract 2019; 15, 6: 326-330

\section{Introduction}

The occurrence of colorectal cancer (CRC) is steadily increasing, making it the third most common malignancy in the world and the second one in Europe [1]. According to data from Surveillance, Epidemiology, and End Results (SEER) colorectal cancer is disseminated in $22 \%$ of patients during the diagnosis [2]. In addition, about 30-40\% of patients in stage III will relapse within five years.
The main treatment method for disseminated CRC is chemotherapy and targeted therapy. The percentage of five-year overall survival (OS) in this group of patients in western countries reaches $14 \%$, which is probably primarily the result of radical surgery in oligometastatic disease (metastasectomy). The median survival for patients receiving fluoropyrimidine, oxaliplatin and irinotecan-based sequential palliative chemotherapy is about 20 months. 
The addition of molecular targeted drugs to the standard mentioned above contributes to the improvement in the treatment results. In cancer cells, about half of patients have activating mutations of the $K R A S$ (exons $2,3,4)$ and $N R A S$ genes (exons 2,3,4), and about $10 \%$ in the $B R A F$ gene [3-5]. These are negative predictors for efficacy of treatment with panitumumab and cetuximab, which are both anti-EGFR (epidermal growth factor receptor) monoclonal antibodies. These medications can be used in mutations-negative patients. Despite different antibody isotypes (panitumumab - IgG2 and cetuximab - IgG1), no differences in their effectiveness were demonstrated [6].

Initially, the benefit from using anti-EGFR antibodies in the third-line therapy was demonstrated in patients with wild-type $K R A S$ tumours - in monotherapy the response was observed in $13 \%$ of patients, and the median OS increased from 4.8 months to 9.5 months compared with best supportive care [7]. Studies assessing the combination of antibodies with first-line chemotherapy not only showed a positive effect on life extension of such treatment, but also enabled the determination of further negative predictive factors as $K R A S$ as well as $N R A S$ and $B R A F$ mutations. The combination of anti-EGFR antibodies with first-line chemotherapy increases the median OS to 26 months and progression-free survival (PFS) to 10 months compared to chemotherapy (20 months and 8 months, respectively) $[8,9]$.

The most common side effects of anti-EGFR antibodies are skin reactions caused by disturbed proliferation, differentiation and migration of keratinocytes as well as stimulation of the non-specific immune response [10]. Symptoms covering skin and its appendages caused by these antibodies are defined as skin toxicity.

\section{Case report}

In February 2018, a 55-year-old female patient with inoperable recurrence of rectal cancer was admitted to the chemotherapy ward to start systemic treatment. The malignancy was primary diagnosed in March 2012 when, due to gastrointestinal obstruction in the course of rectal cancer, a double-barrel sigmoideostomy was performed. Due to extension of the disease the patient underwent induction chemotherapy with capecitabine (04-07.2012; 10.2012-03.2013) and after that she received preoperative chemoradiotherapy (07-08.2012). In May 2013, the patient underwent laparotomy. Intraoperatively, rectal tumour infiltrating the uterus, part of vagina, and the anterior abdominal wall was found. As a consequence low anterior rectal resection by Hartmann method with complete mesorectal resection and hysterectomy was performed. In pathological examination tubular adenocarcinoma G2 ypT2N0 was diagnosed. In September
2013, the patient experienced symptoms of mechanical gastrointestinal obstruction once again. Intraoperatively, intra-abdominal adhesions were found together with the intestinal loop drawn into a massive pelvic inflammatory infiltration, which was probably a late complication of radiation therapy. Ileo-transverse anastomosis was performed. In September 2015, another surgery took place due to vaginal-intestinal fistula symptoms. Loops of the small intestine were dissected distally from the bypass anastomosis, leaving the invaginated stumps.

In November 2017, a positron emission tomography (PET) with 18-fluorodeoxyglucose (18-FDG) was performed. The recurrence of the disease was found in a form of a new lesions - in the bed after complete mesorectal resection (SUV max. FDG 5.2) and peritoneal implants (SUV max. FDG = 6.7).

At admission to the chemotherapy department, the patient was in very good general condition PS0 (PS, performance status) but slightly underweight (BMI 18.9 , body weight $43 \mathrm{~kg}$ ). The patient reported pain in the anal area and loose stools since the last surgery; however, with good appetite, and stable body weight. Physical examination revealed the presence of a massive hernia in the area of the stoma and numerous, movable axillary lymph nodes enlarged to $1 \mathrm{~cm}$. The patient was a smoker. She did not report any chronic diseases. The family history of cancer was negative. In laboratory tests: bone marrow, liver, and kidney functions were normal; there were increased values of carcinoembryonic (CEA, $13.92 \mathrm{ng} / \mathrm{mL}$ ) and carbohydrate antigen (Ca 19-9 antigen; $43.3 \mathrm{U} / \mathrm{mL}$ ).

Due to the lack of $K R A S, N R A S$, and $B R A F$ gene mutations and chronic diarrhea, which could potentially be exacerbated by irinotecan, it was decided to introduce FOLFOX chemotherapy in combination with panitumumab. After the second drug administration she developed a G1 acne-like rash on face and torso, G2 hand-foot syndrome, and G1 diarrhoea. Oral antibiotic therapy with doxycycline at the dose of $2 \times 100 \mathrm{mg}$, topical treatment of skin lesions with vitamin A, E and cholesterol ointment and loperamide $4 \times 4$ mg were introduced. After the $4^{\text {th }}$ cycle, the skin lesions intensified (G2 rash and G3 hand-foot syndrome), so the topical treatment was enriched by $10 \%$ urea ointment. After the $5^{\text {th }}$ cycle, the patient's general condition worsened. The symptoms of short bowel syndrome connected with diarrhoea increased to grade G2 leading to electrolyte disturbances. In addition, trophic changes in the abdominal skin in the area of the stoma (G3) appeared, which made considerable difficulties while taking care of it. It was necessary to prolong the intervals between infusions of cytostatics. Parenteral hydration and correction of electrolyte disturbances were introduced simultaneously with continuation of current symptomatic treatment. Due to the severity of diarrhoea, atropine with diphe- 
noxylate $2.5 \mathrm{mg}+0.025 \mathrm{mg} 3 \times 2$ tablets and morphine $10 \mathrm{mg}$ every four hours were added to the loperamide treatment. A slight improvement was obtained after using racecadotril $100 \mathrm{mg}$ three times a day.

First evaluation of treatment effectiveness, based on the CT scan, revealed cancer stabilisation. However, during this time the patient lost $16 \%$ of baseline body weight (BMI 15.7), and increased her risk associated with nutritional status (NRS, Nutritional Risk Score) which was rated $4-$ as an indication for nutritional treatment. Due to the lack of formal possibilities to return to targeted therapy after discontinuation, it was decided to continue systemic treatment along with symptomatic medications and the use of medical food. In subsequent CT scans, disease stabilization maintained, but clinically due to exacerbation of skin toxicity a temporal brake from panitumumab administration was made. Ointments with hydrocortisone (hydrocortisone, urea $10 \%$, boric acid $1 \%$ ) and gentamicin were added to symptomatic treatment. Further adverse reactions were accompanied by prolonged asymptomatic neutropaenia and G2 peripheral polyneuropathy. During the $16^{\text {th }}$ oxaliplatin infusion a severe allergic reaction occurred. It was manifested by pruritus and redness located on the skin of the limbs.

By January 2019, the patient's PS (PS3) and quality of life deteriorated significantly. Because of this and due to cachexia and unacceptable toxicity treatment was stopped after 16 infusions. For the further care the patient was referred to the Oncological Outpatient Clinic. In a CT scan performed in March 2019, the disease was still stable. The patient died in May 2019, without confirmation of cancer progression.

\section{Discussion}

Skin toxicity most commonly manifested as acne-like rash, periungual inflammation, dryness, and itching of the skin occurs in up to $96 \%$ of patients receiving panitumumab along with chemotherapy. During FOLFOX chemotherapy with panitumumab, skin rash $\mathrm{G} \geq 3$ was observed in $38 \%$ of patients, compared to $2 \%$ of patients receiving chemotherapy alone. However, it has been shown that there are no significant differences in the quality of life of patients depending on whether they have $\mathrm{G} 0-2$ or $\mathrm{G} \geq 3$ skin complications [11]. On the other hand, the development of G2-4 skin toxicity, as opposed to $\mathrm{G} 0-1$, correlates with good response to treatment. In the first group of patients, higher medians of OS and PFS are noted [12-14]. In most patients, skin complications disappear during therapy; however, in some of them, as in the presented case, they persist with significant intensity and may lead to treatment discontinuation.
In this case, skin toxicity was manifested not only as a rash characteristic for anti-EGFR drugs, but also as dryness and trophic changes of the abdominal skin in the area of the stoma and hand-foot syndrome; these additional symptoms significantly impaired patient's quality of life. A skin ulceration in the area of the stoma made sticking an ostomy pouch impossible, which was extremely troublesome due to diarrhoea. In turn, the increased hand-foot syndrome worsened patient's daily activities.

Hand-foot syndrome occurs in $6-64 \%$ of patients treated for cancer, and its frequency depends on the type of systemic treatment used. Erythema, oedema, hyperkeratosis, and signs of dysesthesia usually occur in G1-2 severity. In turn, the G3 degree is associated with flaking skin, as well as blistering and ulceration accompanied by severe pain that significantly affect work and self-service. Hand-foot syndrome is most often a complication of capecitabine, liposomal doxorubicin, docetaxel, or fluorouracil [15]. In the presented case, previous long-term capecitabine chemotherapy was likely to affect the appearance and severity of hand-foot syndrome. In addition, oxaliplatin exacerbated the symptoms of polyneuropathy.

Treatment of skin reactions depends on their severity and includes the use of moisturizers, vitamin $\mathrm{K}$ ointments, protective skin filters (SPF > 15 UVA and UVB), creams containing steroids, and oral antibiotics. If the patient develops a $\mathrm{G} \geq 3$ skin reactions or skin toxicity is deemed unacceptable, it is recommended to suspend anti-cancer drugs temporarily and, after a restoration, continue the therapy in a reduced dose if possible. In some patients, the side effects of anti-EGFR drugs disappear only after permanent discontinuation of therapy.

The strategy for management of this skin toxicity includes not only treatment but also prophylaxis. Currently, prophylactic antibiotic therapy with tetracyclines or with drugs for external use is not a standard procedure because there is no clear evidence of a reduction in skin complications frequency. However, there are reports that such a modification may not only reduce the risk of G2-4 skin toxicity by up to $50 \%$ but is also likely to have a positive impact on the quality of life, mainly during the initial phase of immunotherapy $[16,17]$.

Triple-drug therapy with panitumumab is associated with higher incidence of $\mathrm{G} \geq 3$ diarrhoea (18\% vs. 9\%) [12]. Diarrhoea complicating systemic treatment with panitumumab is known to be less common in patients undergoing prophylactic antibiotic therapy $(56 \%$ $v s .85 \%$ ), which probably results from anti-inflammatory and antibacterial effects of tetracyclines [17].

Numerous anti-diarrhoeal medications were used in the presented case, unfortunately with poor therapeutic effect. Literature indicates that in the described situation some improvement may be achieved by $\mathrm{H} 2$ receptor 
blockers or proton pump inhibitors which reduce diarrhoea in patients with short bowel syndrome [18]. In the presented patient short bowel syndrome was related to both current systemic therapy and previous treatment consisting of surgeries, chemotherapy, and radiation therapy, which led to reduction in length of the intestine and impairment of its function. Short bowel syndrome is defined as a reduction in the absorption of nutrients resulting from excessive shortening or functional disorders of the intestine. It is manifested by debilitating diarrhoea, leading to malnutrition and both water and electrolyte disorders. Treatment of this syndrome should take place in specialist centers. It is based on parenteral nutrition with simultaneous enteral nutrition, aimed at maintaining the function of intestine and preventing atrophy of the intestinal villi.

Limited availability of outpatient clinics that specialize in nutritional treatment is a hindrance to cancer patients. Modification of therapy by adding adequate nutritional treatment is bound to improve the general condition of the patients in whom short bowel syndrome led to cachexia and abandoning chemotherapy.

It is estimated that as many as $10-20 \%$ of cancer patients die due to malnutrition, and only $30-60 \%$ of the ones at risk receive nutritional support (i.e. oral supplements or parenteral nutrition or enteral nutrition). One of the European multicenter studies estimated that nearly $40 \%$ of cancer patients receive incorrect classification of nutrition level, resulting in the lack of adequate nutritional intervention [19].

According to the guidelines of the European Society for Clinical Nutrition and Metabolism (ESPEN), long-term parenteral nutrition should be offered in bowel failure if: 1) enteral nutrition is insufficient, 2) the predicted survival is longer than $2-3$ months, 3 ) it is anticipated that parenteral nutrition may stabilise or improve the patient's performance status and quality of life [20]. In addition, BMI in the range of 18.5$-20.0 \mathrm{~kg} / \mathrm{m}^{2}$ means the patient is at the risk of malnutrition with an indication for the introduction of nutritional treatment. BMI below $18.5 \mathrm{~kg} / \mathrm{m}^{2}$ is diagnosed as malnutrition with the need for urgent nutritional intervention.

Anti-EGFR monotherapy is less likely to cause life-threatening complications in comparison with chemotherapy. However, cumulative toxicity coming from combination of targeted drugs and chemotherapy may impair the quality of life in a significant way. Moreover it may create the need for dose reduction or drug discontinuation which in turn reduces the chances of obtaining desired therapeutic effects. In Poland, cetuximab and panitumumab treatment is financed under the Ministry of Health's drug program. If the antibody used in the first line is discontinued due to toxicity, the drug program does not allow its re-use in the third line even if its ineffectiveness has not been proven. Updated drug program (Novemeber 2019) allows for interruption in cytotoxic treatment but interval between antibody infusions can not be longer than 8 weeks. In this regard, it is extremely important to carry out a proper qualification before administration of triple therapy including anti-EGFR antibodies.

\section{References}

1. Bray F, Ferlay J, Soerjomataram I, et al. Global cancer statistics 2018 GLOBOCAN estimates of incidence and mortality worldwide for 36 cancers in 185 countries. CA Cancer J Clin. 2018; 68(6): 394-424, doi: 10.3322/caac.21492, indexed in Pubmed: 30207593

2. Howlader N, Noone AM, Krapcho M, et al. SEER Cancer Statistics Review, 1975-2016, National Cancer Institute. Bethesda, MD, https:// seer.cancer.gov/csr/1975_2016/, based on November 2018 SEER data submission, posted to the SEER web site, April 2019

3. Xie MZ, Li JL, Cai ZM, et al. Impact of primary colorectal Cancer location on the KRAS status and its prognostic value. BMC Gastroenterol. 2019; 19(1): 46, doi: 10.1186/s12876-019-0965-5, indexed in Pubmed: 30917791.

4. Zhao B, Wang Lu, Qiu H, et al. Mechanisms of resistance to anti-EGFR therapy in colorectal cancer. Oncotarget. 2017; 8(3): 3980-4000, doi: 10.18632/oncotarget.14012, indexed in Pubmed: 28002810.

5. Tran B, Kopetz S, Tie J, et al. Impact of BRAF mutation and microsatellite instability on the pattern of metastatic spread and prognosis in metastatic colorectal cancer. Cancer. 2011; 117(20): 4623-4632, doi: 10.1002/cncr.26086, indexed in Pubmed: 21456008.

6. Price TJ, Peeters M, Kim TW, et al. Panitumumab versus cetuximab in patients with chemotherapy-refractory wild-type KRAS exon 2 metastatic colorectal cancer (ASPECCT): a randomised, multicentre, open-label, non-inferiority phase 3 study. Lancet Oncol. 2014; 15(6): 569-579, doi: 10.1016/S1470-2045(14)70118-4, indexed in Pubmed 24739896.

7. Jonker DJ, Karapetis CS, Harbison C, et al. K-ras mutations and benefit from cetuximab in advanced colorectal cancer. N Engl J Med. 2008; 359(17): 1757-1765, doi: 10.1056/NEJMoa0804385, indexed in Pubmed: 18946061

8. Douillard JY, Oliner KS, Siena S, et al. Panitumumab-FOLFOX4 treatment and RAS mutations in colorectal cancer. N Engl J Med. 2013; 369(11): 1023-1034, doi: 10.1056/NEJMoa1305275, indexed in Pubmed: 24024839

9. Cutsem EV, Lenz HJ, Köhne $\mathrm{CH}$, et al. Fluorouracil, Leucovorin, and Irinotecan Plus Cetuximab Treatment andRASMutations in Colorectal Cancer. J Clin Oncol. 2015; 33(7): 692-700, doi: 10.1200/jco.2014.59.4812.

10. Jost M, Kari C, Rodeck U. The EGF receptor - an essential regulator of multiple epidermal functions. Eur J Dermatol. 2000; 10(7): 505-510, indexed in Pubmed: 11056418

11. Siena S, Tabernero J, Bodoky G, et al. Quality of life during first-line FOLFOX4 \pm panitumumab in wild-type metastatic colorectal carcinoma: results from a randomised controlled trial. ESMO Open. 2016 1(2): e000041, doi: 10.1136/esmoopen-2016-000041, indexed in Pubmed: 27843597.

12. Douillard JY, Siena S, Cassidy J, et al. Final results from PRIME: randomized phase III study of panitumumab with FOLFOX4 for first-line treatment of metastatic colorectal cancer. Ann Oncol. 2014; 25(7): 1346-1355, doi: 10.1093/annonc/mdu141, indexed in Pubmed: 24718886 .

13. Saltz LB, Meropol NJ, Loehrer PJ, et al. Phase II trial of cetuximab in patients with refractory colorectal cancer that expresses the epidermal growth factor receptor. J Clin Oncol. 2004; 22(7): 1201-1208, doi: 10.1200/JCO.2004.10.182, indexed in Pubmed: 14993230.

14. Douillard JY Rong A Sidhu R. RAS mutations in colorectal cancer. $N$ Engl J Med. 2013; 369(22): 2159-2160, doi: 10.1056/NEJMc1312697.

15. Kowalska M, Kowalik A, Góźdź S. Dermatologic adverse events associated with chemotherapy and targeted anticancer therapy. Przeglad Dermatologiczny. 2016; 2: 127-138, doi: 10.5114/dr.2016.59135.

16. Ocvirk J, Heeger S, McCloud P, et al. A review of the treatment options for skin rash induced by EGFR-targeted therapies: Evidence from randomized clinical trials and a meta-analysis. Radiol Oncol. 2013; 47(2): 166-175, doi: 10.2478/raon-2013-0014, indexed in Pubmed: 23801914 
17. Lacouture ME, Mitchell EP, Piperdi B, et al. Skin toxicity evaluation protocol with panitumumab (STEPP), a phase II, open-label, randomized trial evaluating the impact of a pre-Emptive Skin treatment regimen on skin toxicities and quality of life in patients with metastatic colorectal cancer. J Clin Oncol. 2010; 28(8): 1351-1357, doi: 10.1200/JCO.2008.21.7828, indexed in Pubmed 20142600.

18. Pironi L, Arends J, Bozzetti F et al. Home Artificial Nutrition \& Chronic Intestinal Failure Special Interest Group of ESPEN, Home Artificial Nutrition \& Chronic Intestinal Failure Special Interest Group of ESPEN.
ESPEN guidelines on chronic intestinal failure in adults. Clin Nutr. 2016; 35(2): 247-307, doi: 10.1016/j.clnu.2016.01.020, indexed in Pubmed: 26944585

19. Arends J, Baracos $V$, Bertz $H$, et al. ESPEN expert group recommendations for action against cancer-related malnutrition. Clin Nutr. 2017; 36(5): 1187-1196, doi: 10.1016/j.clnu.2017.06.017, indexed in Pubmed: 28689670

20. Bozzetti F, Arends J, Lundholm K, et al. ESPEN quidelines on parenteral nutrition: non-surgical oncology. Clinical Nutrition. 2009; 28(4): 445-454, doi: 10.1016/j.clnu.2009.04.011. 